\title{
Generalized seniority with realistic interactions in open-shell nuclei
}

\author{
M A Caprio ${ }^{1}$, F Q Luo ${ }^{1}, \mathrm{~K} \mathrm{Cai}^{1,2} \ddagger$, Ch Constantinou ${ }^{1}$ \\ and V Hellemans ${ }^{1,3}$ \\ ${ }^{1}$ Department of Physics, University of Notre Dame, Notre Dame, Indiana \\ 46556-5670, USA \\ 2 Department of Physics, Bard College, Annandale-on-Hudson, New York \\ 12504-5000, USA \\ ${ }^{3}$ Physique Nucléaire Théorique et Physique Mathématique, Université Libre de \\ Bruxelles, CP229, B-1050 Brussels, Belgium
}

\begin{abstract}
Generalized seniority provides a truncation scheme for the nuclear shell model, based on pairing correlations, which offers the possibility of dramatically reducing the dimensionality of the nuclear shell-model problem. Systematic comparisons against results obtained in the full shell-model space are required to assess the viability of this scheme. Here, we extend recent generalized seniority calculations for semimagic nuclei, the $\mathrm{Ca}$ isotopes, to open-shell nuclei, with both valence protons and valence neutrons. The even-mass $\mathrm{Ti}$ and $\mathrm{Cr}$ isotopes are treated in a full major shell and with realistic interactions, in the generalized seniority scheme with one broken proton pair and one broken neutron pair. Results for level energies, orbital occupations, and electromagnetic observables are compared with those obtained in the full shellmodel space. We demonstrate that, even for the Ti isotopes, significant benefit would be obtained in going beyond the approximation of one broken pair of each type, while the $\mathrm{Cr}$ isotopes require further broken pairs to provide even qualitative accuracy.

PACS numbers: 21.60.Cs,21.60.Ev
\end{abstract}

\section{Introduction}

The generalized seniority scheme $[1,2]$, or broken pair approximation $[3,4]$, has long been proposed as a truncation scheme for the nuclear shell model, based on pairing correlations, with the potential to dramatically reduce the dimensionality of the nuclear shell-model problem. The generalized seniority scheme has also been proposed as a microscopic foundation for the phenomenologically successful interacting boson model (IBM) [5], through the Otsuka-Arima-Iachello mapping [6,7]. The underlying premise of the generalized seniority scheme is that the ground state of an even-even nucleus can be well approximated by a condensate built from collective $S$ pairs. These are defined as a specific linear combination of pairs of nucleons in the different valence orbitals, $\ddagger$ Present address: Perimeter Institute for Theoretical Physics, Waterloo, Ontario N2L 2Y5, Canada. 
each pair coupled to angular momentum zero. A shell-model calculation for the ground state and low-lying states can then be carried out in a truncated space, consisting of states built from a condensate of collective $S$ pairs together with a small number $v$ (the generalized seniority) of nucleons not forming part of an $S$ pair.

Although the generalized seniority approach has been applied in various contexts [820], only recently has it been benchmarked against calculations carried out in the full shell-model space, with realistic interactions. Comparisons have so far focused on semimagic nuclei [21-23]. Systematic comparisons for both even-mass and odd-mass Ca isotopes, across the full $p f$ shell $(20 \leq N \leq 40)$, with the FPD6 [24] and GXPF1 [25] interactions, are presented in [23]. These benchmark calculations for semimagic nuclei are based on the assumption of at most one broken pair, i.e., $v=2$ for even-mass isotopes, which is found to provide a quantitatively successful reproduction of many of the full-space results (energies, occupations, and electromagnetic observables) for the lowest-lying (and, in particular, yrast) states. In the interior of the shell, where both valence protons and neutrons are present, the obvious challenge to the generalized seniority truncation is the seniority-nonconserving, or pair-breaking, nature of the proton-neutron quadrupole-quadrupole interaction [26]. As this interaction induces deformation, it also suppresses pairing correlations.

The purpose of the present work is to investigate the viability of a highly-truncated generalized seniority description as one moves beyond semimagic nuclei, introducing valence nucleons of both types, and to map the breakdown of this description. The generalized seniority scheme, as a simple representation of the full nuclear shell-model problem, is foremost of conceptual interest. The generalized seniority model space of generalized seniority $v$ is equivalent to the space spanned by BCS states with at most $v$ quasiparticles, projected onto definite particle number. Therefore, the principal question being addressed by the present calculations is to what extent the shell-model wave functions and predictions quantitatively reflect a simple structure based on BCSlike pairing, in a form which nonetheless fully accounts for particle number conservation. However, the generalized seniority scheme may also be of computational relevance, as a practical truncation scheme. In the $p f$ shell, the dimensions of the full shell-model space for semimagic and near-semimagic nuclei are computationally tractable. However, truncation is still necessary if single-particle spaces significantly larger than the $p f$ shell are considered. A generalized seniority truncation is more likely to be advantageous for weakly-deformed nuclei near closed-shell, in large single-particle spaces, than for strongly-deformed nuclei with large numbers of both protons and neutrons in the valence shell.

We extend the investigations of the Ca isotopes $(Z=20)[23]$ into the interior of the $p f$ shell, to the even-even $\mathrm{Ti}(Z=22)$ and $\operatorname{Cr}(Z=24)$ isotopes, establishing a benchmark comparison of results obtained in the generalized seniority truncation against results obtained in the full shell-model space. We consider calculations truncated to one broken proton pair plus one broken neutron pair, i.e., $\left(v_{p}, v_{n}\right)=(2,2)$. These calculations should be viewed as a baseline, in that they are based on the most restricted 
generalized seniority truncation for both protons and neutron. Calculations involving more broken pairs could be expected to provide a description of the dynamics which extends further into the interior of the shell. The present study is therefore also intended to provide an indication of the dependences upon valence proton and neutron numbers, interactions, and observables likely to influence successful treatment of nuclei in such spaces of higher generalized seniority.

The generalized seniority basis, in a proton-neutron scheme, and computational method are summarized in section 2. Then, calculations for the $\mathrm{Ti}$ and $\mathrm{Cr}$ isotopes in the generalized seniority scheme are compared with full shell-model calculations, for level energies (section 3.1), orbital occupations (section 3.2), and electromagnetic observables (section 3.3). Preliminary results were presented in [27].

\section{Generalized seniority scheme}

We briefly review the construction of the generalized seniority basis for like particles [4, $10,23,28]$ and its extension to a proton-neutron scheme. Let $C_{a, m_{a}}^{\dagger}$ be the creation operator for a particle in the shell-model orbital $a \equiv\left(n_{a} l_{a} j_{a}\right)$, with angular momentum projection quantum number $m_{a}$. Then the collective $S$ pair of the generalized seniority scheme is defined by $S^{\dagger} \equiv \sum_{a} \frac{1}{2} \alpha_{a} \hat{\jmath}_{a}\left(C_{a}^{\dagger} C_{a}^{\dagger}\right)^{(0)}$, where $a$ runs over the active orbitals, and $\hat{\jmath}_{a} \equiv\left(2 j_{a}+1\right)^{1 / 2}$. This operator creates a linear combination of pairs in different orbitals $a$, with amplitudes $\alpha_{a}$.

A basis state within the generalized seniority scheme then consists of a "condensate" of collective pairs, together with $v$ additional nucleons not forming part of a collective $S$ pair. The number $v$ is termed the generalized seniority. Thus, if we consider semimagic nuclei, so only like valence particles (all neutrons or all protons) are present, the $v=0$ condensate state is constructed as $S^{\dagger \mathcal{N}}|\rangle$, with angular momentum $J=0$. States with $v=2$ and angular momentum $J$ are then obtained as $S^{\dagger \mathcal{N}-1}\left(C_{a}^{\dagger} C_{b}^{\dagger}\right)^{(J)}|\rangle$, states with $v=4$ as $S^{\dagger \mathcal{N}-2}\left[\left(C_{a}^{\dagger} C_{b}^{\dagger}\right)^{\left(J_{12}\right)}\left(C_{c}^{\dagger} C_{d}^{\dagger}\right)^{\left(J_{34}\right)}\right]^{(J)}|\rangle$, etc., where, if $n$ is the number of valence nucleons, $\mathcal{N}=n / 2$ is the total number of pairs.

To construct a generalized seniority basis, the parameters $\alpha_{a}$ must first be determined. This is usually accomplished variationally, so as to minimize the energy functional $E_{\alpha}=\left\langle S^{\mathcal{N}}|H| S^{\mathcal{N}}\right\rangle /\left\langle S^{\mathcal{N}} \mid S^{\mathcal{N}}\right\rangle[3,29]$, subject to the conventional normalization $\sum_{a}\left(2 j_{a}+1\right) \alpha_{a}^{2}=\sum_{a}\left(2 j_{a}+1\right)[10]$. The $S$-condensate state has the same form as a number-projected BCS ground state, and these $\alpha_{a}$ coefficients are related to BCS occupancy parameters $u_{a}$ and $v_{a}$ by $\alpha_{a}=v_{a} / u_{a}$ [4]. The generalized seniority states as defined above are in general unnormalized and nonorthogonal (for $v \geq 3$, they also form an overcomplete set). However, a suitable basis is obtained by a Gram-Schmidt procedure, which yields orthonormal basis states as linear combinations of the original states, e.g., for $v=2$,

$$
|\mathcal{N} ; v=2 ; J, k\rangle=\sum_{a b} c_{a b ; J k}\left|S^{\mathcal{N}-1}\left(C_{a} C_{b}\right)^{(J)}\right\rangle
$$

where $k$ is simply a counting index for the orthogonalized states. Several approaches [4, 


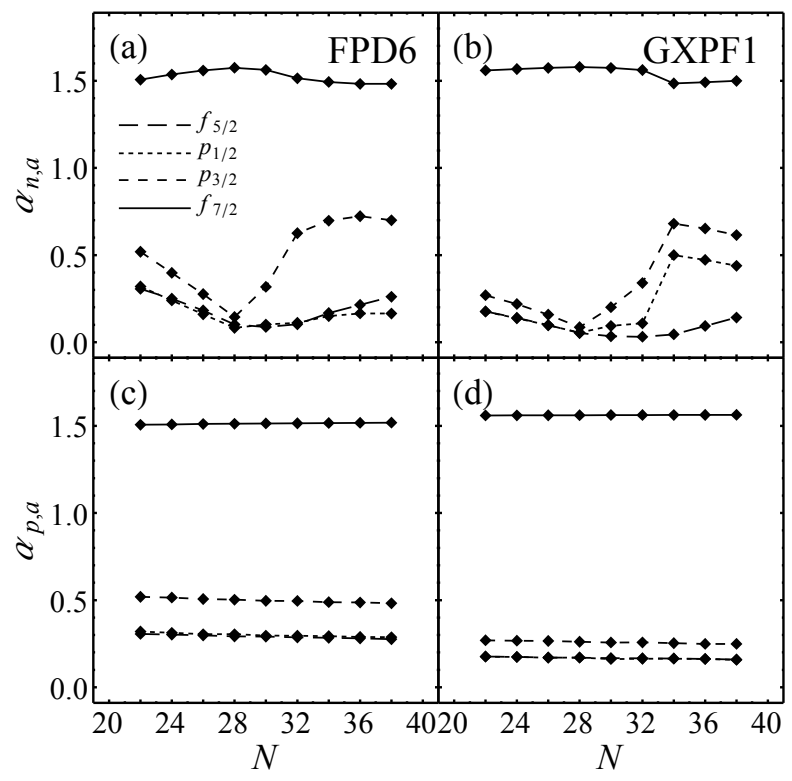

Figure 1. Structure of the collective $S$ pairs for $\mathrm{Ti}$, for neutrons (top) and protons (bottom), and for the FPD6 (left) and GXPF1 (right) interactions. Amplitudes are obtained by the variational prescription (see text).

$13,28,30,31]$ have been developed for evaluating matrix elements of one-body and twobody operators in the generalized seniority basis. The present calculations make use of recurrence relations derived in [32], where the notation and methods used in the present work are also established in detail.

A generalized seniority basis can be defined for nuclei with valence particles of both types via a proton-neutron scheme, that is, by taking all possible products of proton and neutron generalized seniority states, with generalized seniorities $v_{p}$ and $v_{n}[4,19]$. Here we consider states with one broken pair of each type $\left[\left(v_{p}, v_{n}\right)=(2,2)\right]$ and thus have basis states

$$
\left[\left|\mathcal{N}_{p} ; v_{p}=2 ; J_{p}, k_{p}\right\rangle \otimes\left|\mathcal{N}_{n} ; v_{n}=2 ; J_{n}, k_{n}\right\rangle\right]^{(J)} .
$$

The coefficients $\alpha_{p, a}$ appearing in the proton collective pair operator $\left[S_{p}^{\dagger} \equiv\right.$ $\left.\sum_{a} \frac{1}{2} \alpha_{p, a} \hat{\jmath}_{a}\left(C_{p, a}^{\dagger} C_{p, a}^{\dagger}\right)^{(0)}\right]$ and the coefficients $\alpha_{n, a}$ appearing in the neutron collective pair operator $\left[S_{n}^{\dagger} \equiv \sum_{a} \frac{1}{2} \alpha_{n, a} \hat{\jmath}_{a}\left(C_{n, a}^{\dagger} C_{n, a}^{\dagger}\right)^{(0)}\right]$ are distinct, and these are chosen independently, by minimizing $E_{p, \alpha_{p}}=\left\langle S_{p}^{\mathcal{N}_{p}}|H| S_{p}^{\mathcal{N}_{p}}\right\rangle /\left\langle S_{p}^{\mathcal{N}_{p}} \mid S_{p}^{\mathcal{N}_{p}}\right\rangle$ and $E_{n, \alpha_{n}}=\left\langle S_{n}^{\mathcal{N}_{n}}|H| S_{n}^{\mathcal{N}_{n}}\right\rangle /\left\langle S_{n}^{\mathcal{N}_{n}} \mid S_{n}^{\mathcal{N}_{n}}\right\rangle$, respectively. The values of the amplitudes $\alpha_{p, a}$ and $\alpha_{n, a}$ obtained in this manner for the Ti isotopes are shown in figure 1, for both the FPD6 and GXPF1 interactions. The variational expression $E_{p, \alpha_{p}}$ for the proton $S$ pair has no manifest dependence upon neutron number and, similarly, the variational expression $E_{n, \alpha_{n}}$ for the neutron $S$ pair has no manifest dependence upon proton number. However, an implicit dependence is induced, in each case, by the $A$-dependent definitions of the interaction two-body matrix elements, which are proportional to $A^{-0.35}$ for FPD6 [24] or $A^{-0.3}$ for GXPF1 [25]. A simple multiplicative scaling of the Hamiltonian would not affect the collective pair 


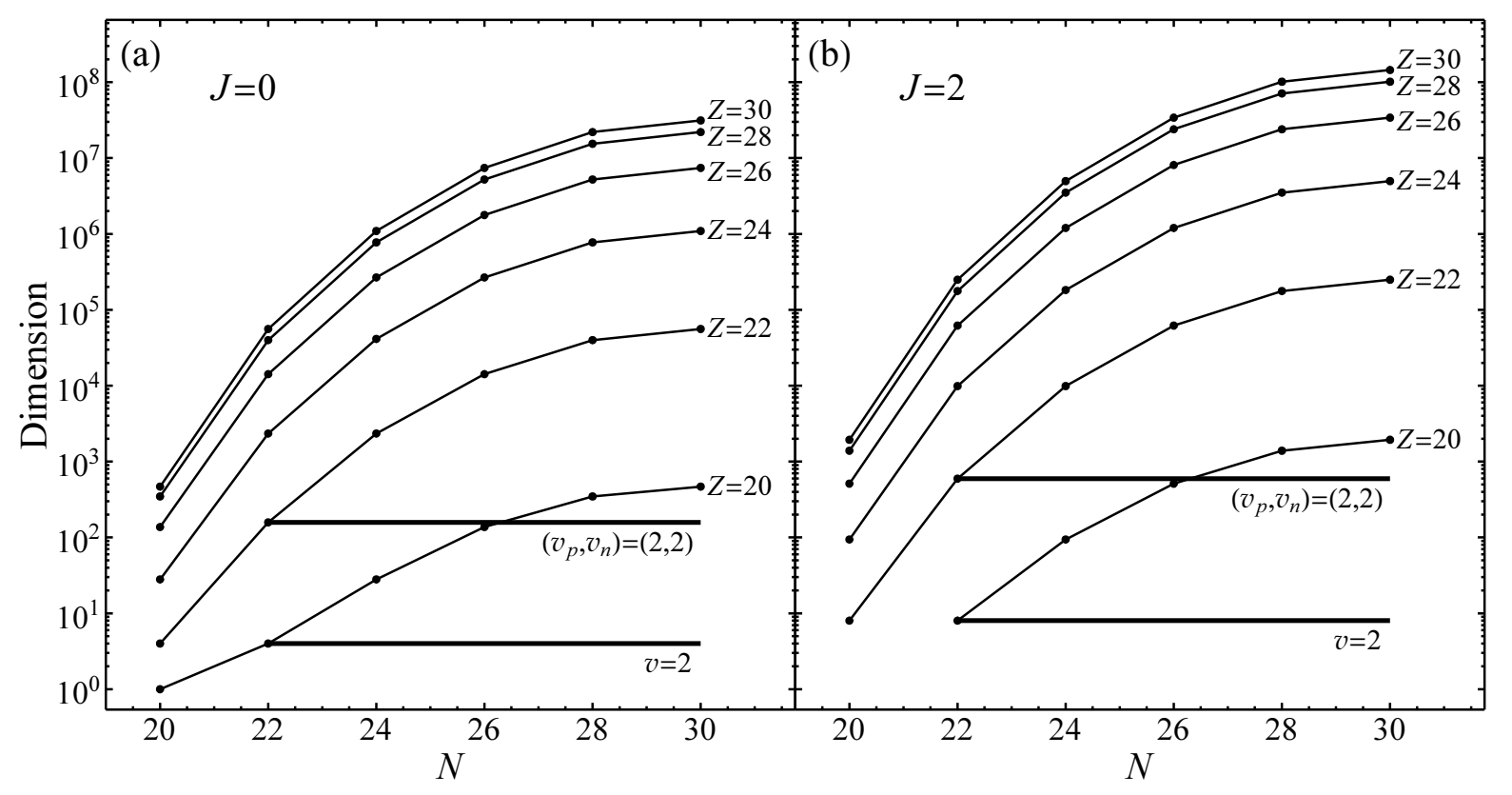

Figure 2. Dimensions of the $J$-scheme shell-model spaces for nuclei in the $p f$ shell, with $20 \leq N, Z \leq 30$, shown for (a) $J=0$ and (b) $J=2$. The dimension of the generalized seniority model space with one broken pair $(v=2)$ is shown for comparison with the semimagic $\mathrm{Ca}$ isotopes, and the dimension of the generalized seniority model space with one broken pair of each type $\left[\left(v_{p}, v_{n}\right)=(2,2)\right]$ is shown for comparison with open-shell nuclei. Dimensions for nuclei with $30<N, Z \leq 40$ follow by particle-hole conjugation symmetry.

structure, but note that here rather the $A$-dependent two-body contribution varies in strength relative to the $A$-independent single-particle energies. Consequently, the values of $\alpha_{n, a}$ in the shell interior vary slightly $(\lesssim 1 \%)$ between successive isotopic chains, and the values of $\alpha_{p, a}$ vary slightly along each isotopic chain [figure 1 (bottom)].

For semimagic nuclei, the size of the generalized seniority basis is the same as for the shell-model problem with only $v$ particles in the same set of orbitals, regardless of the number of $S$ pairs. Thus, the generalized seniority model space with one broken pair $(v=2)$ for any even Ca isotope taken in the $p f$ shell has the same dimension as the shell-model space for ${ }^{42} \mathrm{Ca}$ - dimension 4 for $J=0,8$ for $J=2,6$ for $J=4$, etc. [23]. Similarly, if both valence protons and neutrons are present, the basis size is the same as for the shell-model problem with only $v_{p}$ protons and $v_{n}$ nucleons. Thus, the generalized seniority model space with one broken proton pair and one broken neutron pair $\left[\left(v_{p}, v_{n}\right)=(2,2)\right]$ for any even-even nucleus taken in the $p f$ shell has the same dimension as the shell-model space for ${ }^{44} \mathrm{Ti}$ - dimension 158 for $J=0,596$ for $J=2$, 655 for $J=4$, etc. For comparison, the dimensions of the full shell-model spaces in the $p f$ shell are shown, in the $J$ scheme, in figure 2. The reduction in model space dimension from the full shell-model space to the generalized seniority truncated space considered in this work exceeds two orders of magnitude for the Ti isotopes midshell and approaches four orders of magnitude for the Cr isotopes midshell (e.g., factors of 
$\sim 4 \times 10^{2}$ and $\sim 7 \times 10^{3}$ in the $J=0$ spaces for ${ }^{52} \mathrm{Ti}$ and ${ }^{54} \mathrm{Cr}$, respectively).

\section{Results}

\subsection{Energies}

Here we consider some basic energy observables, for the even-even $\mathrm{Ti}(Z=22)$ and $\mathrm{Cr}$ $(Z=24)$ isotopes, in the generalized seniority $\left(v_{p}, v_{n}\right)=(2,2)$ space. Calculations are shown for $20<N<40$, carried out in the full $p f$ shell $\left(f_{7 / 2}, p_{3 / 2}, p_{1 / 2}\right.$, and $f_{5 / 2}$ orbitals) for both protons and neutrons, with the FPD6 [24] and GXPF1 [25] interactions. The generalized seniority results are benchmarked against those obtained in the full shellmodel space, calculated using the code NuSHELLX [33].

We begin by considering the energy eigenvalue for the $J=0$ ground state. The calculated values are summarized in figure 3 (left). The ground state energy is shown as obtained both for the $\left(v_{p}, v_{n}\right)=(0,0)$ proton-neutron $S$-pair condensate and in the $\left(v_{p}, v_{n}\right)=(2,2)$ generalized seniority model space, as well as in the full shell-model space.

To provide a baseline for comparison, it is helpful to review the analogous results for the semimagic Ca isotopes in the $v=2$ space, from [23], shown at top in figure 3. It should first be noted (see appendix of [23]) that the ground state obtained in the $v=2$ space is simply the $v=0 S$-pair condensate, provided the $\alpha_{a}$ coefficients have been chosen variationally as in section 2 . Therefore, the ground-state energies obtained in $v=0$ and $v=2$ spaces are identical. [In contrast, for nuclei in the interior of the shell, the ground state obtained in the $\left(v_{p}, v_{n}\right)=(2,2)$ space is not in general the $\left(v_{p}, v_{n}\right)=(0,0) S$-pair condensate, and these states are considered separately in

figure 3.] The ground state energies [figure 3(a,b)] obtained for $\mathrm{Ca}$ in the generalized seniority truncation are essentially indistinguishable from those obtained in the full shell-model space, when viewed on the $\sim 100 \mathrm{MeV}$ scale of the valence shell interaction energies. A more useful measure of the level of agreement is provided by the residual energy difference $\Delta E$, shown in figure 3 (right), obtained by subtracting the full space result from the generalized seniority result. This difference may be thought of as the missing correlation energy, not accounted for by the generalized seniority description of the ground state. For the Ca isotopes [figure $3(\mathrm{~g}, \mathrm{~h})$ ], the maximum difference occurs approximately midshell, peaking at $0.48 \mathrm{MeV}$ for the FPD6 interaction or $0.21 \mathrm{MeV}$ for the GXPF1 interaction. It is worth noting at this point that the deviations between the generalized seniority results and the full-space results were found to be consistently smaller for the GXPF1 interaction than for the FPD6 interaction in the study of Ca isotopes - not just for the ground state energy, but for excitation energies, orbital occupations (section 3.2), and electromagnetic observables (section 3.3), as well.

Considering now the ground-state eigenvalues for $\mathrm{Ti}$ in the generalized seniority scheme [figure $3(\mathrm{c}, \mathrm{d})$ ], a $\left(v_{p}, v_{n}\right)=(0,0)$ calculation, which simply takes the expectation value of the Hamiltonian in the proton-neutron $S$ pair condensate, misses $\sim 2-6 \mathrm{MeV}$ 

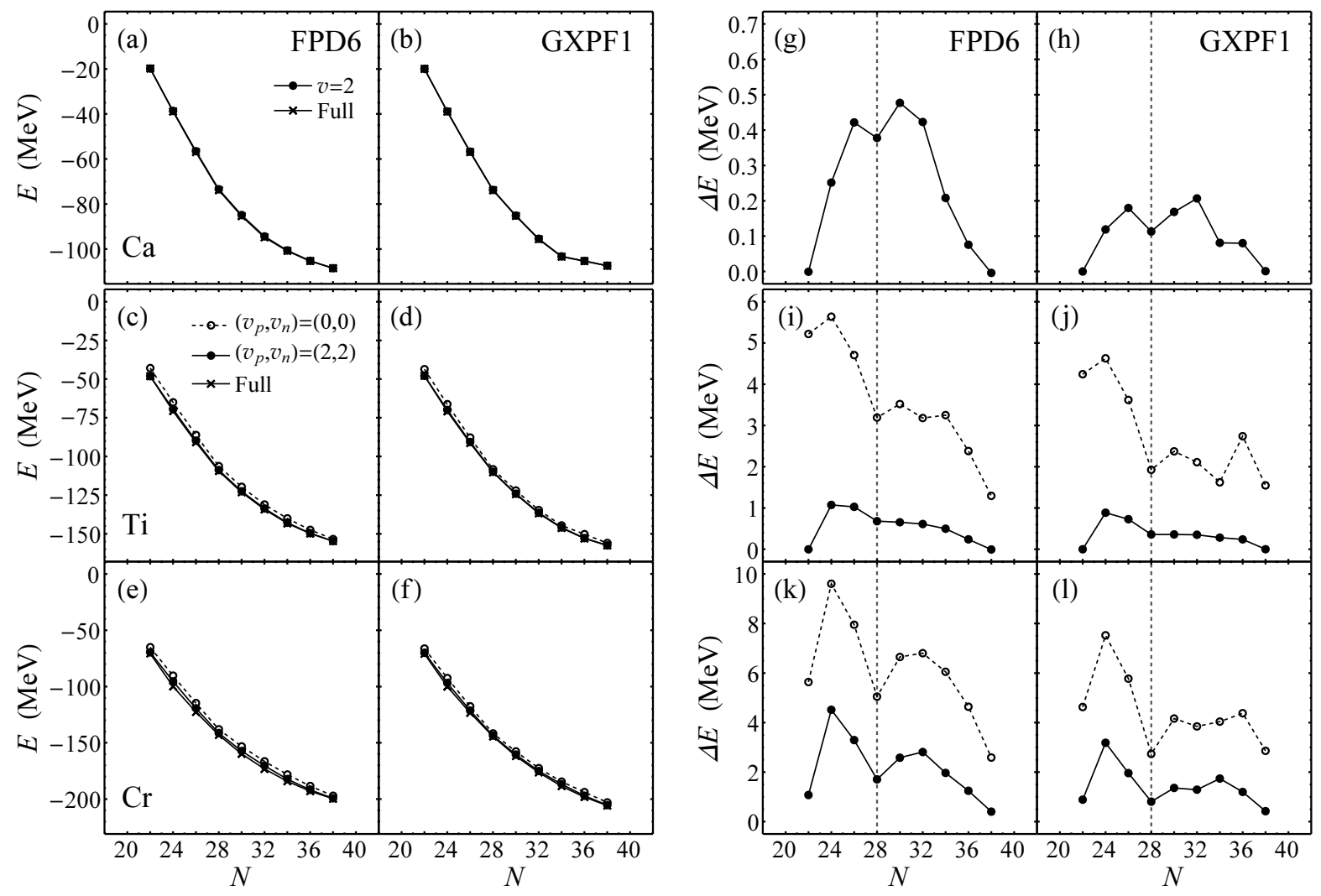

Figure 3. (Left) Energy eigenvalue of the $0^{+}$ground state, calculated in the generalized seniority $S$-pair condensate (open circles), $\left(v_{p}, v_{n}\right)=(2,2)$ model space (or $v=2$ for Ca) (solid circles) and full shell-model space (crosses). (Right) Residual energy difference $\Delta E$ of the generalized seniority result relative to the full shell-model result. Values are shown for the even-mass $\mathrm{Ca}$, $\mathrm{Ti}$, and $\mathrm{Cr}$ isotopes (top to bottom, respectively), for the FPD6 and GXPF1 interactions. The dashed vertical line indicates the $N=28$ subshell closure. Calculations for Ca are from [23].

in binding energy. However, the $\left(v_{p}, v_{n}\right)=(2,2)$ model space accounts for most of the missing correlation energy, leaving a difference of $\lesssim 1 \mathrm{MeV}$ between the generalized seniority and full-space results [figure $3(\mathrm{i}, \mathrm{j})]$. Since Ti has two valence protons in the $p f$ shell, a $\left(v_{p}, v_{n}\right)=(2,2)$ calculation encompasses the full space of proton configurations. Moreover, for $N=22\left({ }^{44} \mathrm{Ti}\right)$ and $N=38\left({ }^{60} \mathrm{Ti}\right)$, there are only two valence neutrons or neutron holes, so the $\left(v_{p}, v_{n}\right)=(2,2)$ space is in fact identical to the full shellmodel space, and the generalized seniority and full shell-model results are strictly identical. The generalized seniority results lie furthest from the full-space results in the middle of the $f_{7 / 2}$ subshell $(20<N<28)$, with a maximum deviation of $1.1 \mathrm{MeV}$ for $N=24\left({ }^{46} \mathrm{Ti}\right)$ with the FPD6 interaction or $0.9 \mathrm{MeV}$ with the GXPF1 interaction. The generalized seniority results more closely match the full-space results in the upper pf shell $(28<N<40)$, where the average deviations are $0.47 \mathrm{MeV}$ for the FPD6 interaction or $0.28 \mathrm{MeV}$ for the GXPF1 interaction.

For the evolution of $\mathrm{Cr}$ ground-state eigenvalues across the shell [figure 3(e,f)], 
Table 1. Average residual energy differences $\Delta E$ (in $\mathrm{MeV}$ ) of the energy eigenvalues calculated in the generalized seniority $\left(v_{p}, v_{n}\right)=(2,2)$ model space (or $v=2$ for Ca) relative to the full shell-model results, for selected states of the $\mathrm{Ca}, \mathrm{Ti}$, and $\mathrm{Cr}$ isotopes, and for the FPD6 and GXPF1 interactions. These are root-mean-square averages over the full set of even-mass isotopes with $20<N<40$. Calculations for Ca are from [23].

\begin{tabular}{cccccccccc}
\hline & \multicolumn{4}{c}{ FPD6 } & & \multicolumn{4}{c}{ GXPF1 } \\
\cline { 2 - 4 } \cline { 7 - 9 } & $0_{1}^{+}$ & $2_{1}^{+}$ & $4_{1}^{+}$ & $0_{2}^{+}$ & & $0_{1}^{+}$ & $2_{1}^{+}$ & $4_{1}^{+}$ & $0_{2}^{+}$ \\
\hline $\mathrm{Ca}$ & 0.31 & 0.48 & 0.62 & 0.88 & & 0.13 & 0.25 & 0.34 & 0.63 \\
$\mathrm{Ti}$ & 0.65 & 1.10 & 1.14 & 2.68 & & 0.45 & 0.76 & 0.82 & 1.66 \\
$\mathrm{Cr}$ & 2.48 & 4.54 & 5.07 & 5.74 & & 1.62 & 3.04 & 3.55 & 4.02 \\
\hline
\end{tabular}

similar qualitative observations apply as to the $\mathrm{Ti}$ isotopes. However, the energy differences between the generalized seniority and full shell-model results are much larger, up to $\sim 10 \mathrm{MeV}$ for the $\left(v_{p}, v_{n}\right)=(0,0)$ calculation. Breaking one pair of each type yields a less dramatic improvement than observed for the Ti isotopes. The generalized seniority results $\left(v_{p}, v_{n}\right)=(2,2)$ again lie furthest from the full-space results in the middle of the $f_{7 / 2}$ subshell, missing $4.5 \mathrm{MeV}$ of binding energy for $N=24\left({ }^{48} \mathrm{Cr}\right)$ with FPD6 or 3.2 MeV with GXPF1. The generalized seniority results again more closely match the full-space results in the upper $p f$ shell, where the average deviations are $2.0 \mathrm{MeV}$ for the FPD6 interaction or $1.3 \mathrm{MeV}$ for the GXPF1 interaction. It has been hypothesized by Monnoye et al. [19] that the generalized seniority description should improve at subshell closures. The residual energy difference does indeed have a sharp local minimum at $N=28$, for both interactions, in both the $S$-condensate $\left[\left(v_{p}, v_{n}\right)=(0,0)\right]$ and brokenpair $\left[\left(v_{p}, v_{n}\right)=(2,2)\right]$ calculations.

In considering excitation energies, we focus on the low-lying states, for which the generalized seniority description was found to be most successful in the semimagic nuclei, especially the first $2^{+}$and $4^{+}$states. The succussful reproduction of the properties of the first excited $0^{+}$state requires a greater number of broken pairs, especially in the $f_{7 / 2}$ subshell [23]. For the energy eigenvalues of the ground state and other low-lying states, the average (root-mean-square) deviations of the generalized seniority $\left(v_{p}, v_{n}\right)=(2,2)$ model space results from the full shell-model results across the shell are summarized in table 1 , along with the corresponding $v=2$ results for Ca.

The excitation energies $E_{x}$ of the first $2^{+}$and $4^{+}$states, calculated relative to the $0^{+}$ground state, are shown in figure 4 . We again begin by reviewing the results for the Ca isotopes, at top in figure 4 . The broad features of the evolution of excitation energies across the shell are reproduced within the $v=2$ model space. For instance, for the $2^{+}$ state [figure $4(\mathrm{a}, \mathrm{b})$ ], spikes in excitation energy are obtained at the $f_{7 / 2}$ subshell closure $(N=28)$ and $p_{3 / 2}$ subshell closure $(N \approx 32-34)$. The excitation energy calculated for the $2^{+}$state deviates from that calculated in the full model space by at most $0.41 \mathrm{MeV}$ for FPD6 or $0.23 \mathrm{MeV}$ for GXPF1. The deviations in excitation energy for the $4^{+}$state 

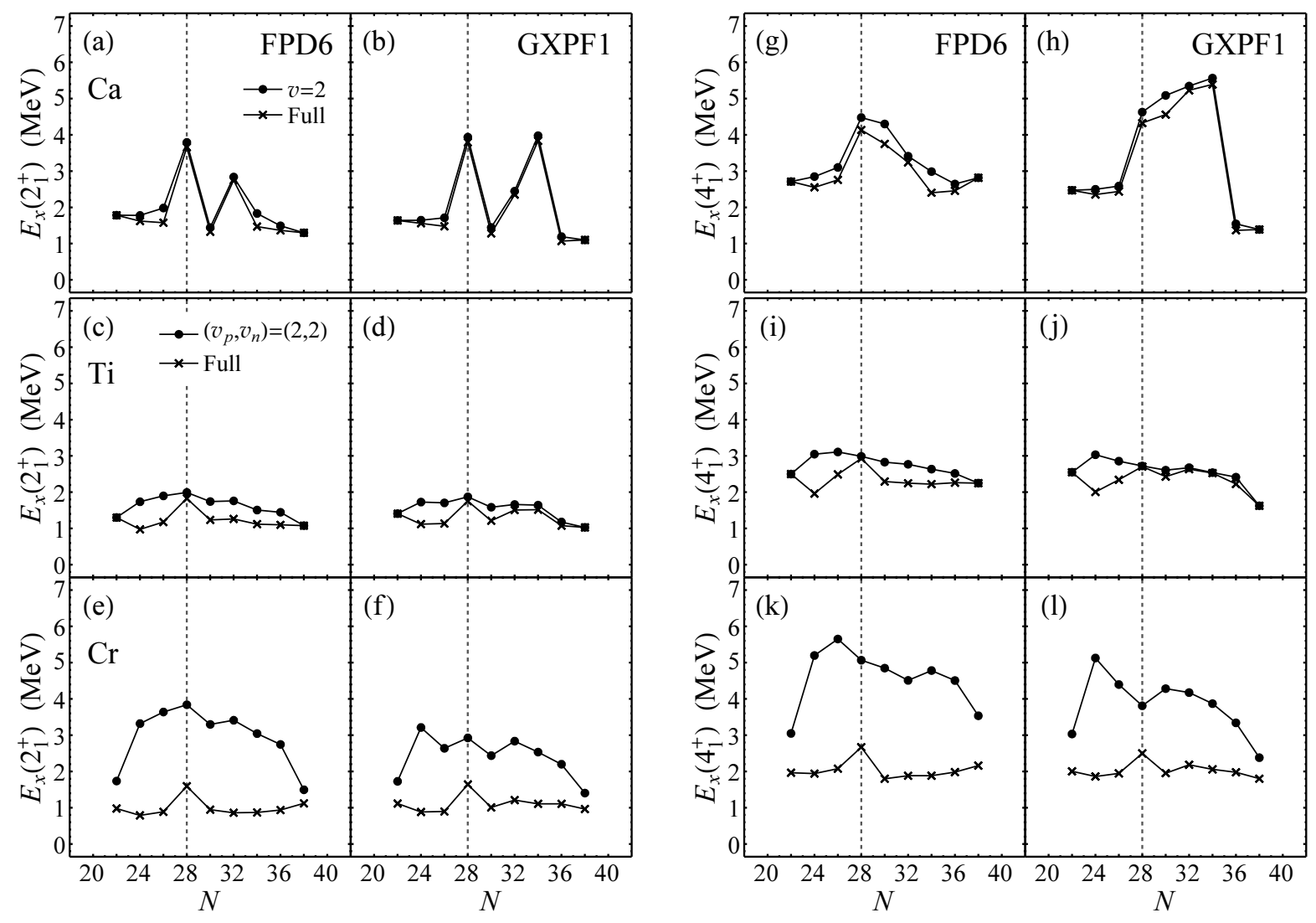

Figure 4. Excitation energies $E_{x}$ of the first $2^{+}$(left) and $4^{+}$(right) states, calculated in the generalized seniority $\left(v_{p}, v_{n}\right)=(2,2)$ model space (or $v=2$ for Ca) (circles) and full shell-model space (crosses). Values are shown for the even-mass $\mathrm{Ca}$, Ti, and Cr isotopes (top to bottom, respectively), for the FPD6 and GXPF1 interactions. The dashed vertical line indicates the $N=28$ subshell closure. Calculations for Ca are from [23].

[figure $4(\mathrm{~g}, \mathrm{~h})$ ] are only modestly larger, at most $0.58 \mathrm{MeV}$ for FPD6 or $0.53 \mathrm{MeV}$ for GXPF1.

For the excitation energies of the $2^{+}$[figure $\left.4(\mathrm{c}, \mathrm{d})\right]$ and $4^{+}$[figure $\left.4(\mathrm{i}, \mathrm{j})\right]$ states in the $\mathrm{Ti}$ isotopes, the values obtained in the generalized seniority truncated space and the full shell-model space necessarily agree at $N=22$ and $N=38$, where, as noted above, the $\left(v_{p}, v_{n}\right)=(2,2)$ space is identical to the full space. However, otherwise, substantial differences arise. It is interesting to note the cases in which these calculations at low generalized seniority are most successful. Differences are largest in the $f_{7 / 2}$ subshell. Then, at the subshell closure $(N=28)$, the excitation energies calculated in the generalized seniority truncated space closely reproduce those in the full space - moderately closely for the $2^{+}$energy, to $0.17 \mathrm{MeV}$ for FPD6 and $0.12 \mathrm{MeV}$ for GXPF1, and much more closely for the $4^{+}$energy, to $0.060 \mathrm{MeV}$ (or $\sim 2 \%$ ) for FPD6 and $0.017 \mathrm{MeV}$ (or $\sim 0.6 \%$ ) for GXPF1. In the upper $p f$ shell, specifically for the GXPF1 interaction, the excitation energies of the $2^{+}$[figure $4(\mathrm{~d})$ ] and $4^{+}$states [figure $4(\mathrm{j})$ ] are reproduced with a quantitative accuracy comparable to that observed in 
the semimagic nuclei. Deviations from the full shell-model results average only $0.19 \mathrm{MeV}$ for the $2^{+}$state or $0.12 \mathrm{MeV}$ for the $4^{+}$state. The excitation energies obtained in the generalized seniority truncated model space are systematically higher than those in the full model space, i.e., more correlation energy is missed in the excited state eigenvalue than in the ground state eigenvalue.

For the Cr isotopes, it is seen that considering only one broken pair of each type is markedly inadequate for description of the excitation energies, for both the $2^{+}$[figure $\left.4(\mathrm{e}, \mathrm{f})\right]$ and $4^{+}$[figure $4(\mathrm{k}, \mathrm{l})$ ] states. The actual excitation energies calculated in the full space are comparable to those for the Ti isotopes, but the excitation energies obtained in the generalized seniority truncation are about twice as high.

\subsection{Occupations}

The occupations of orbitals provide a direct measure of the structure of an eigenstate, here as obtained either in a generalized seniority calculation or the full shell-model space. Unlike conventional shell-model basis states, the generalized seniority basis states do not have definite occupation for each orbital, rather, involving a BCS-like distribution of occupations. Therefore, the occupation $\left\langle n_{a}\right\rangle$ of an orbital $a=\left(n_{a} l_{a} j_{a}\right)$ in an eigenstate represented in this basis cannot be obtained as a simple average over the contributing basis states, but rather must be evaluated as the expectation value of a one-body operator, the number operator for the orbital $\left[n_{a}=-\hat{\jmath}_{a}\left(C_{a}^{\dagger} \tilde{C}_{a}\right)^{(0)}\right]$, by the process described in $[23,32]$. Occupations of each of the $p f$-shell orbitals in the $0^{+}$ ground state are shown in figure 5 , for the $\mathrm{Ca}$, $\mathrm{Ti}$, and $\mathrm{Cr}$ isotopes, both for neutron orbitals [figure 5 (left)] and proton orbitals [figure 5 (right)].

We again begin by reviewing the situation for the $\mathrm{Ca}$ isotopes [figure 5(a,b)]. Recall that the ground state obtained in the $v=2$ space, for semimagic nuclei, is simply the $v=0 S$-pair condensate (section 3.1). For $20 \leq N \leq 28$, the neutrons are almost exclusively found in the $f_{7 / 2}$ orbital. Therefore, the generalized seniority scheme essentially reduces to conventional seniority in a single $j$-shell. However, in the upper $p f$ shell, for $28<N<40$, neutrons fill multiple orbitals simultaneously, and the generalized seniority scheme prescribes correlations among these $j$-shells beyond the conventional seniority correlations within each $j$-shell. The generalized seniority calculations closely reproduce the occupations obtained in the full shell-model space, to within 0.1 nucleon across the entire shell, and with deviations averaging only 0.03 nucleon for FPD6 or 0.015 for GXPF1. The occupations obtained in the first $2^{+}$and $4^{+}$states follow very similar patterns to those for the ground state. For the first excited $0^{+}$state, the occupations are only well-described in the upper $p f$ shell (see figure 5 of [23]) - for $N<28$, the single-particle energies strongly favor an excitation involving promotion of a single particle from the $f_{7 / 2}$ orbital to the $p_{3 / 2}$ orbital, which is not supported in the highly-restricted (dimension 4) $J=0, v=2$ space.

Let us consider now the occupations of these same neutron orbitals for the $0^{+}$ground state of the $\mathrm{Ti}$ isotopes [figure $5(\mathrm{c}, \mathrm{d})]$, as obtained in the $\left(v_{p}, v_{n}\right)=(2,2)$ generalized 

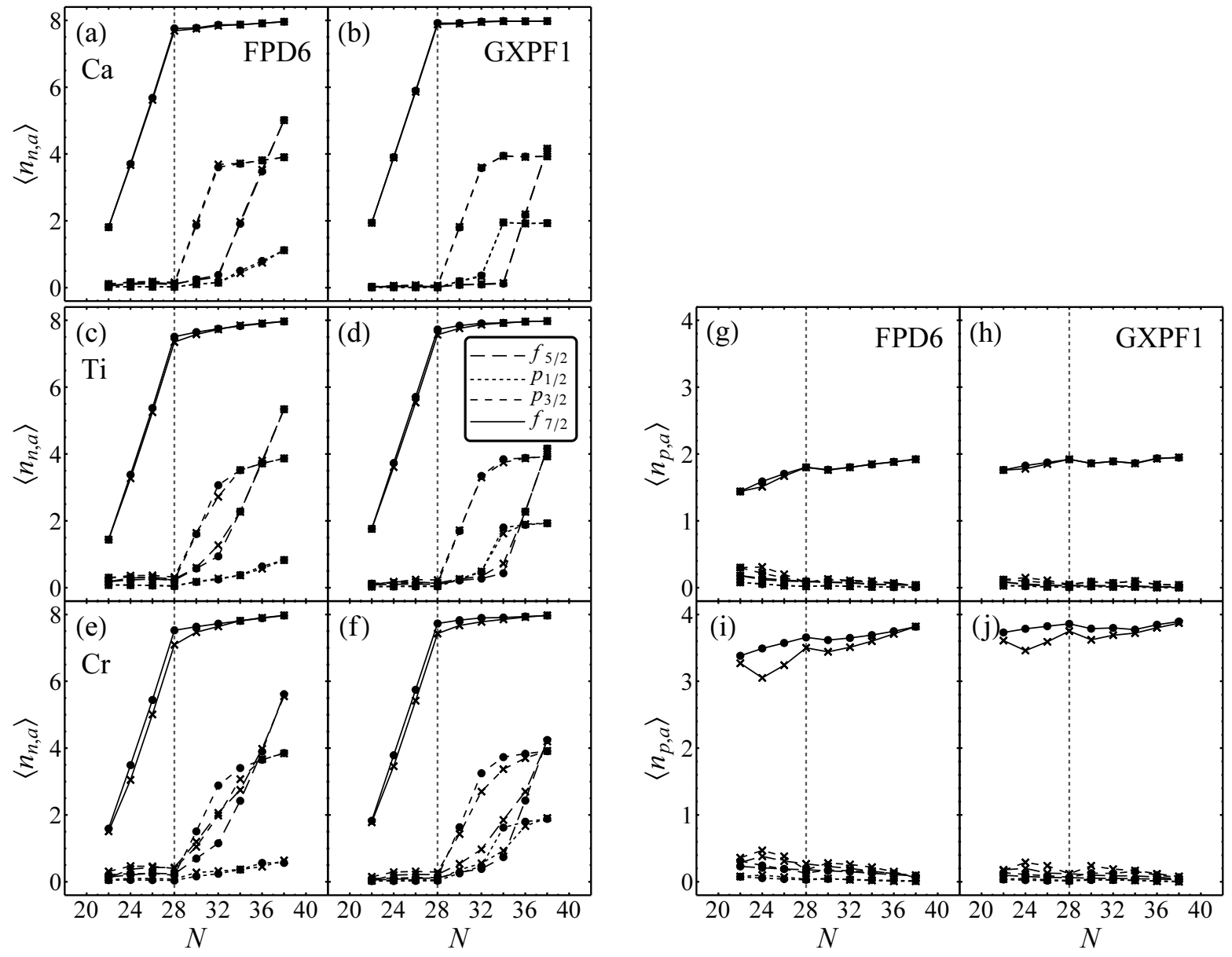

Figure 5. Orbital occupations for the $0^{+}$ground state, for neutron (left) and proton (right) orbitals, calculated in the generalized seniority $\left(v_{p}, v_{n}\right)=(2,2)$ model space (or $v=2$ for Ca) (circles) and full shell-model space (crosses). Values are shown for the even-mass $\mathrm{Ca}$, $\mathrm{Ti}$, and $\mathrm{Cr}$ isotopes (top to bottom, respectively), for the FPD6 and GXPF1 interactions. The dashed vertical line indicates the $N=28$ subshell closure. Calculations for Ca are from [23].

seniority space. The occupations are reproduced to within 0.3 nucleon throughout the shell, with deviations averaging 0.10 nucleon for FPD6 or 0.08 for GXPF1 greater than for the semimagic nuclei, but still leaving the generalized seniority and full-space curves in figure 5(c,d) largely indistinguishable. The deviations which do arise are localized in $N$ and are seen to follow a systematic pattern. Over most of the shell, but especially around the $f_{7 / 2}$ subshell closure at $N=28$, the generalized seniority calculation overestimates the occupation of the neutron $f_{7 / 2}$ orbital. Similarly, at the nominal $p_{3 / 2}$ subshell closure at $N=32$, the generalized seniority calculations overestimate the $p_{3 / 2}$ occupation relative to the $f_{5 / 2}$ occupation. In this limited set of examples, the generalized seniority calculations would appear to favor a filling order more strictly reflecting the ordering of single-particle energies $\left(f_{7 / 2}, p_{3 / 2}, p_{1 / 2}, f_{5 / 2}\right)$ than is actually found in the solutions in the full shell-model space. It is worth noting that 

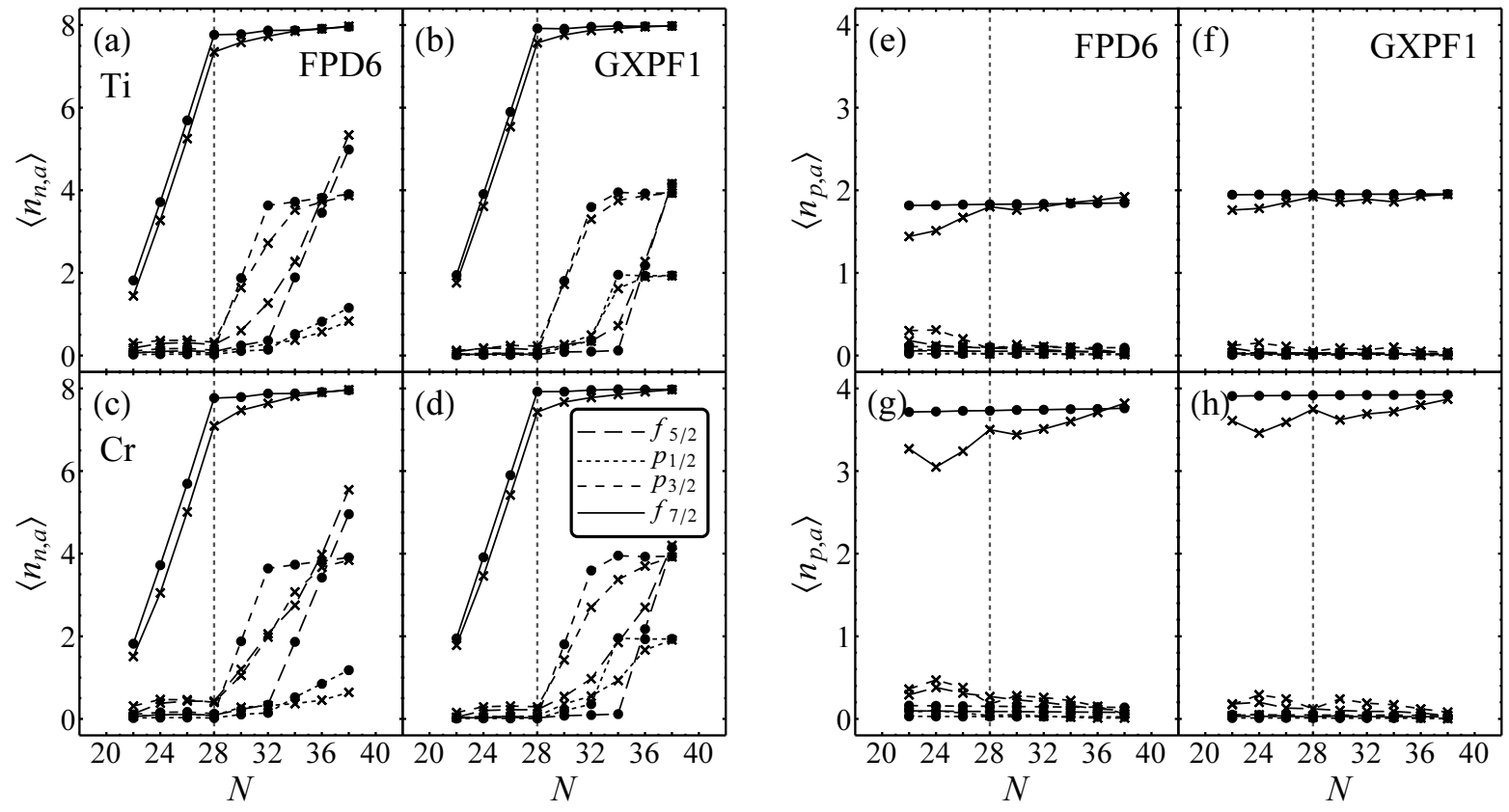

Figure 6. Orbital occupations for the $0^{+}$ground state described as the $\left(v_{p}, v_{n}\right)=(0,0)$ proton-neutron $S$-pair condensate (circles) - for comparison with those obtained in the $\left(v_{p}, v_{n}\right)=(2,2)$ model space in figure 5 - for the even $\mathrm{Ti}$ and $\mathrm{Cr}$ isotopes (top to bottom, respectively), for neutron (left) and proton (right) orbitals, and for the FPD6 and GXPF1 interactions. Occupations obtained in the full shell-model space (crosses) are shown again as well. The dashed vertical line indicates the $N=28$ subshell closure.

the quantitative accuracy obtained for the Ti occupations in figure 5(c,d) is obtained only through the breaking of collective $S$ pairs. For comparison, occupations calculated in the $\left(v_{p}, v_{n}\right)=(0,0)$ proton-neutron $S$-pair condensate for the Ti isotopes are shown in figure $6(\mathrm{a}, \mathrm{b})$. These do reflect the overall evolution of the neutron occupations with $N$, but with substantially larger deviations, averaging 0.31 nucleon for FPD6 or 0.20 nucleon for GXPF1.

For the $\mathrm{Cr}$ isotopes [figure $5(\mathrm{e}, \mathrm{f})$ ], the accuracy with which the neutron orbital occupations for the $0^{+}$ground state are reproduced within the generalized seniority truncation deteriorates, averaging 0.30 nucleon for both FPD6 and GXPF1. The systematic deviations continue to follow the trend described above for the Ti isotopes in which the generalized seniority results follow nominal filling order more strictly than the full shell-model results - but now increased in magnitude, extending over larger ranges of neutron number, and observed in an overestimate of the $p_{1 / 2}$ orbital occupation relative to $f_{5 / 2}$ as well.

In both the $\mathrm{Ti}$ and $\mathrm{Cr}$ isotopes, occupations obtained in the generalized seniority scheme for the first $2^{+}$and $4^{+}$states follow very similar patterns to those for the ground state, and with a comparable (or marginally lower) level of accuracy, summarized in table 2. Occupations for the first excited $0^{+}$state are obtained with comparable accuracy to that obtained in the semimagic nuclei, and, in $\mathrm{Cr}$, comparable to that 
Table 2. Average deviations of neutron orbital occupations $\left\langle n_{n, a}\right\rangle$ calculated in the generalized seniority $\left(v_{p}, v_{n}\right)=(2,2)$ model space (or $v=2$ for $\mathrm{Ca}$ ) relative to the full shell-model results, for selected states of the $\mathrm{Ca}$, $\mathrm{Ti}$, and $\mathrm{Cr}$ isotopes, and for the FPD6 and GXPF1 interactions. These are root-mean-square averages over the full set of even-mass isotopes with $20<N<40$, taking all four $p f$-shell orbitals into account. Calculations for Ca are from [23].

\begin{tabular}{cccccccccc}
\hline & \multicolumn{4}{c}{ FPD6 } & & \multicolumn{4}{c}{ GXPF1 } \\
\cline { 2 - 6 } \cline { 7 - 9 } & $0_{1}^{+}$ & $2_{1}^{+}$ & $4_{1}^{+}$ & $0_{2}^{+}$ & & $0_{1}^{+}$ & $2_{1}^{+}$ & $4_{1}^{+}$ & $0_{2}^{+}$ \\
\hline $\mathrm{Ca}$ & 0.03 & 0.09 & 0.11 & 0.29 & & 0.015 & 0.03 & 0.08 & 0.26 \\
$\mathrm{Ti}$ & 0.10 & 0.18 & 0.17 & 0.37 & & 0.08 & 0.13 & 0.09 & 0.30 \\
$\mathrm{Cr}$ & 0.30 & 0.34 & 0.36 & 0.27 & & 0.30 & 0.35 & 0.42 & 0.24 \\
\hline
\end{tabular}

for the lower-lying states as well (table 2). This is perhaps surprising, given the much poorer description of energies for the excited $0^{+}$state in the generalized seniority scheme (table 1).

The occupations of the proton orbitals along the $\mathrm{Ti}$ and $\mathrm{Cr}$ isotopic chains, by the two valence protons for $\mathrm{Ti}[$ figure $5(\mathrm{~g}, \mathrm{~h})$ ] or four for $\mathrm{Cr}$ [figure $5(\mathrm{i}, \mathrm{j})$ ], depend on the filling of the neutron orbitals. In the full shell-model space, these protons have a significant probability of occupying orbitals other than the $f_{7 / 2}$ orbital, especially for $N \lesssim 28$. As noted in section 2 , the $\alpha_{p, a}$ coefficients in the proton $S$ pair are nearly constant along an isotopic chain [figure 1 (bottom)]. Therefore, the proton occupations obtained in a simple $S$-pair condensate, shown in figure 6 (right), are likewise nearly constant along the chain, varying by only $\sim 1 \%-2 \%$ for the $\mathrm{Ti}$ and $\mathrm{Cr}$ isotopes. However, for the $\mathrm{Ti}$ isotopes, the $\left(v_{p}, v_{n}\right)=(2,2)$ generalized seniority space [figure $5(\mathrm{~g}, \mathrm{~h})$ ] largely accounts for the actual variation in the proton $f_{7 / 2}$ orbital occupation, to within 0.08 nucleon throughout the shell. The agreement is significantly better for $28<N<40$, to within 0.007 nucleon, with differences from the full-space results averaging only 0.0046 nucleons for FPD6 or 0.0039 nucleons for GXPF1. The nature of the deviation is, as for the neutron orbitals, to overestimate the occupation of the $f_{7 / 2}$ orbital. For the Cr isotopes [figure $5(\mathrm{i}, \mathrm{j})$ ], the deviations follow the same pattern, but with significantly larger magnitudes.

\subsection{Electromagnetic observables}

Electromagnetic moments and transition strengths probe correlations in the eigenstates which are not simply apparent from the occupations of section 3.2. Of particular interest is the extent to which electric quadrupole correlations are reproduced in a space truncated to low generalized seniority, as one moves into the shell interior, where the proton-neutron quadrupole-quadrupole interaction is expected to play an increasingly significant role. The electric quadrupole reduced transition probability $B\left(E 2 ; 2_{1}^{+} \rightarrow 0_{1}^{+}\right)$ and quadrupole moment $Q\left(2_{1}^{+}\right)$are shown for the $\mathrm{Ca}$, $\mathrm{Ti}$, and $\mathrm{Cr}$ isotopes in figure 7 . 

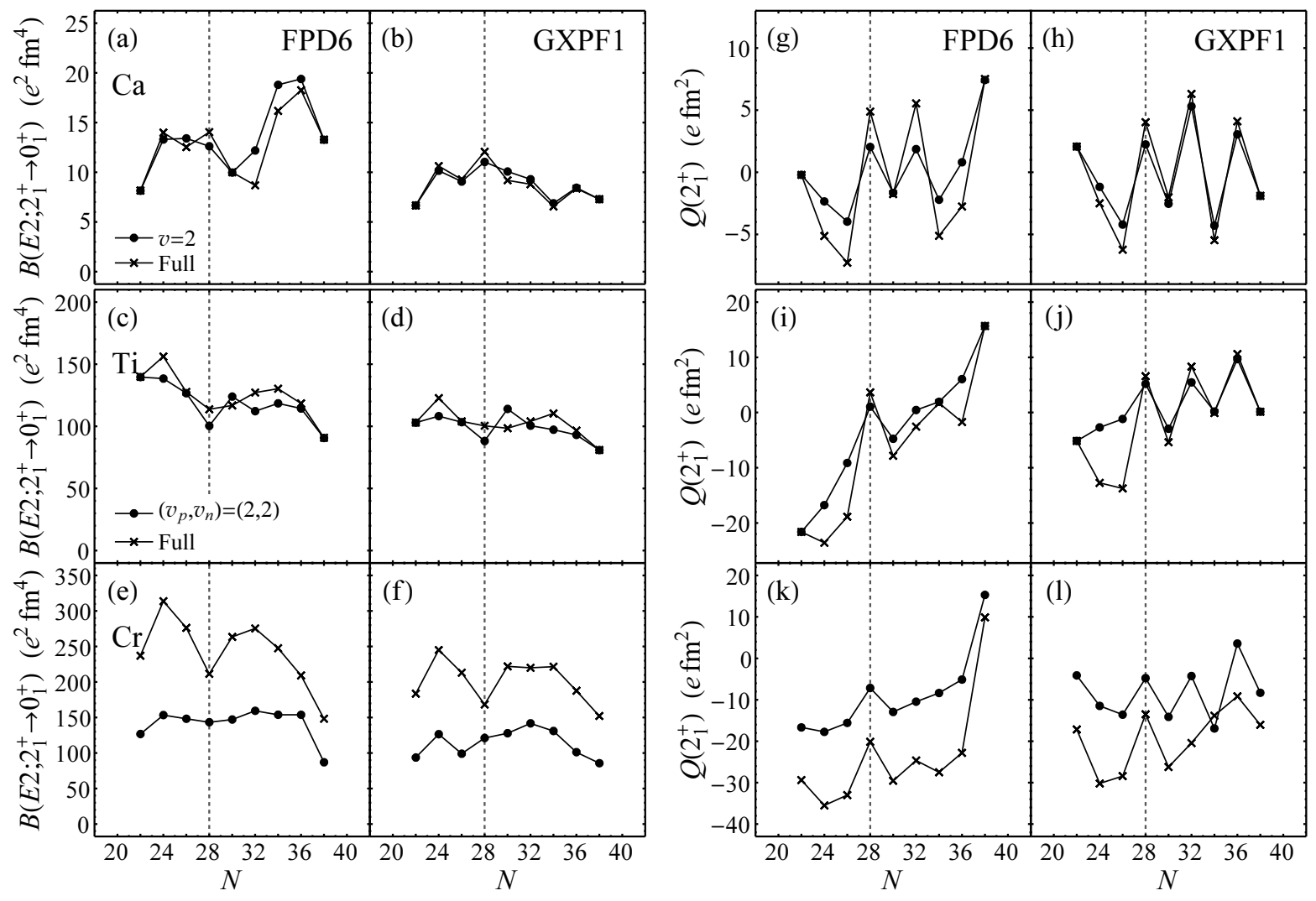

Figure 7. Electric quadrupole observables $B\left(E 2 ; 2_{1}^{+} \rightarrow 0_{1}^{+}\right)$(left) and $Q\left(2_{1}^{+}\right)$(right), calculated in the generalized seniority $\left(v_{p}, v_{n}\right)=(2,2)$ model space (or $v=2$ for Ca) (circles) and full shell-model space (crosses). Values are shown for the even-mass $\mathrm{Ca}$, $\mathrm{Ti}$, and $\mathrm{Cr}$ isotopes (top to bottom, respectively), for the FPD6 and GXPF1 interactions, and are obtained using effective charges $e_{p}=1.5$ and $e_{n}=0.5$. The dashed vertical line indicates the $N=28$ subshell closure. Calculations for Ca are from [23].

Electromagnetic transition matrix elements are obtained from the one-body densities, which are evaluated in the generalized seniority scheme as described in $[23,32]$.

To review the situation for the Ca isotopes [23], the evolution of $B\left(E 2 ; 2_{1}^{+} \rightarrow 0_{1}^{+}\right)$ strengths [figure $7(\mathrm{a}, \mathrm{b})$ ] across the shell is reproduced in the generalized seniority $v=2$ space in its qualitative features (minima and maxima) and with a quantitative accuracy averaging $\sim 12 \%$ for FPD6 or $\sim 6 \%$ for GXPF1. The magnitude of $Q\left(2_{1}^{+}\right)$[figure $7(\mathrm{~g}, \mathrm{~h})$ ] is markedly attenuated in the generalized seniority calculations relative to the full-space results, although the generalized seniority results demonstrate the same qualitative features as the results in the full space, notably exhibiting the same alternations in sign as a function of $N$.

For the Ti isotopes, the $B\left(E 2 ; 2_{1}^{+} \rightarrow 0_{1}^{+}\right)$strengths [figure $7(\mathrm{c}, \mathrm{d})$ ] are an order of magnitude larger than for the Ca isotopes [note the change of scale from figure $7(\mathrm{a}, \mathrm{b})$ to figure $7(\mathrm{c}, \mathrm{d})$ ]. The variation with neutron number is relatively flat, but downsloping with increasing $N$. Both these broad characteristics are well-reproduced in the generalized 


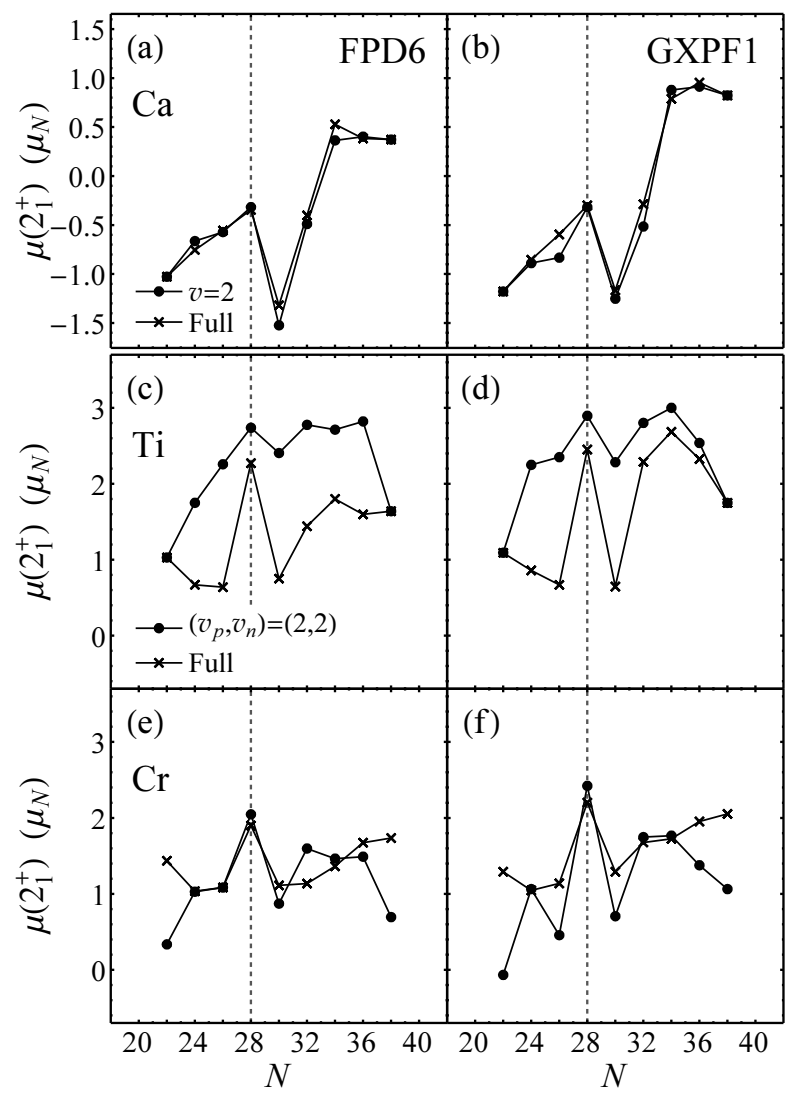

Figure 8. Magnetic moment $\mu\left(2_{1}^{+}\right)$, calculated in the generalized seniority $\left(v_{p}, v_{n}\right)=$ $(2,2)$ model space (or $v=2$ for Ca) (circles) and full shell-model space (crosses). Values are shown for the even-mass $\mathrm{Ca}, \mathrm{Ti}$, and $\mathrm{Cr}$ isotopes (top to bottom, respectively), for the FPD6 and GXPF1 interactions, and are obtained using free-space $g$-factors. The dashed vertical line indicates the $N=28$ subshell closure. Calculations for Ca are from [23].

seniority $\left(v_{p}, v_{n}\right)=(2,2)$ space, although the generalized seniority calculations do not reproduce the detailed fluctuations with neutron number. The deviations under FPD6 are $\sim 8 \%$ [averaging $10 e^{2} \mathrm{fm}^{4}$, on $B(E 2)$ values averaging $126 e^{2} \mathrm{fm}^{4}$ ] or under GXPF1 $\sim 9 \%$ [averaging $9.4 e^{2} \mathrm{fm}^{4}$, on $B(E 2)$ values averaging $103 e^{2} \mathrm{fm}^{4}$ ]. In contrast, the quadrupole moments [figure $7(\mathrm{i}, \mathrm{j})$ ] are poorly reproduced in the $\left(v_{p}, v_{n}\right)=(2,2)$ calculations, even qualitatively, below the $N=28$ subshell closure. They are somewhat better reproduced above the subshell closure, in particular for the GXPF1 interaction, where the detailed variation with $N$ is reproduced. The deviations in this range $28<N<40$ are $\sim 50 \%$ for FPD6 [averaging $4.0 e^{2} \mathrm{fm}^{4}$, on $B(E 2)$ values averaging $8.0 e^{2} \mathrm{fm}^{4}$ ] but a somewhat smaller $\sim 27 \%$ for GXPF1 [averaging $1.7 e^{2} \mathrm{fm}^{4}$, on $B(E 2)$ values averaging $\left.6.5 e^{2} \mathrm{fm}^{4}\right]$.

The $B\left(E 2 ; 2_{1}^{+} \rightarrow 0_{1}^{+}\right)$strengths, as obtained in the full shell-model space, approximately double moving from the $\mathrm{Ti}$ isotopes to the $\mathrm{Cr}$ isotopes [figure 7(e,f)]. However, the $B(E 2)$ strengths in the generalized seniority calculations appear to have saturated, with minimal increase relative to the Ti isotopes. The magnitudes of for the 
quadrupole moments [figure $7(\mathrm{k}, \mathrm{l})$ ] for the Cr isotopes are similarly underestimated by a factor of approximately two in the $\left(v_{p}, v_{n}\right)=(2,2)$ space, although the $N$-dependence is more complicated. Thus, considering only one broken pair of each type fails to produce meaningful results for electric quadrupole observables for the $\mathrm{Cr}$ isotopes.

The magnetic dipole moments $\mu\left(2_{1}^{+}\right)$, shown in figure 8 , are well-reproduced for the Ca isotopes in the generalized seniority $v=2$ space [figure $8(a, b)$ ]. However, for the Ti [figure 8(c,d)] and Cr [figure 8(e,f)] isotopes, the dipole moment calculated in the $\left(v_{p}, v_{n}\right)=(2,2)$ space has an $N$-dependence which bears little qualitative resemblance to that of the full shell-model results. The best reproduction is right at the neutron $N=28$ subshell closure and, as found above for other observables, in the upper $p f$-shell for the Ti isotopes with the GXPF1 interaction.

\section{Conclusions}

The success of calculations truncated at low generalized seniority, here involving one broken proton pair and one broken neutron pair, depends on several factors. As anticipated, the $p n$ interaction precipitates a breakdown of the viability of such a severe truncation with respect to generalized seniority, which is found to retain some applicability two protons away from closed-shell ( $\mathrm{Ti}$ ) but not four protons away from closed-shell (Cr). Comparing results obtained with FPD6 and GXPF1, it is seen that the particular interaction plays a significant role. It would be valuable to have a systematic understanding of how this dependence might reflect underlying quantitative properties of the interaction. As noted in [23], a decomposition of the interaction into an appropriate set of pairing and nonpairing (e.g., quadrupole) components through spectral distribution theory, as in [34], could yield relevant measures. The relative scales of the single-particle energy differences and the two-body interaction strengths may also play a role [35]. The presence of a subshell closure also has significant implications for the success of the generalized seniority approximation, as previously proposed by Monnoye et al. [19]. Between subshell closures, the filling order of orbitals within the shell seems to be influential, i.e., whether there is effectively a single active $j$-shell, as for $20 \leq N \leq 28$, or several $j$-shells are being filled simultaneously, as in the upper $p f$ shell. The low generalized seniority approximations are found to miss the most binding energy, in the present examples, for nuclei with $N \approx Z$, e.g., ${ }^{48} \mathrm{Cr}$, suggesting alternatively that missing $T=0$ pairing correlations may be responsible for some of the deviations observed in the $f_{7 / 2}$ nuclei. These correlations might be more fully accomodated in the isospin generalized seniority scheme [36] than in the proton-neutron scheme.

The present studies serve as a baseline for a possible improved generalized seniority description, not simply through the inclusion of more broken pairs, but, more fundamentally, through selection of the collective proton and neutron $S$ pairs. For an ideal generalized seniority conserving interaction in semimagic nuclei, as considered by Talmi [1], the pair amplitudes would be determined statically, by diagonalization in the two-particle space, and would be constant across the shell. The variational 


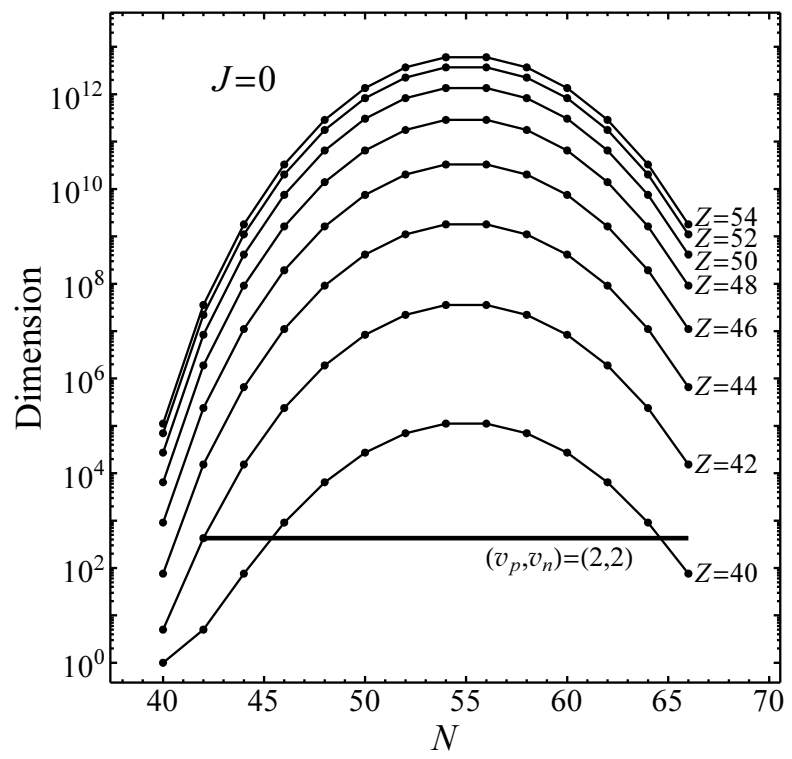

Figure 9. Dimensions of the $J=0$ shell-model spaces for nuclei treated in the $s d g$ major shell. The dimension of the generalized seniority $\left(v_{p}, v_{n}\right)=(2,2)$ model space is shown for comparison. Dimensions for nuclei with $55<N, Z \leq 70$ follow from those with $40 \leq N, Z<55$ by particle-hole conjugation symmetry.

prescription $[3,4]$ used here to determine the pair amplitudes is a dynamical prescription, in that it considers the effects of $p p$ interactions in determining the proton amplitudes and $n n$ interactions in the determining the neutron amplitudes. However, the deviations in calculated occupancies, as noted in section 3.2, are systematic and smoothlyvarying. This suggests that it may be possible to determine the $S$-pair amplitudes which in turn determine the occupancies in the $S$-pair condensate and thus the starting point for improvement with broken pairs - more optimally. For instance, an alternative prescription for obtaining the collective pair amplitudes by angularmomentum projection of the Hartree-Fock-Bogoliubov intrinsic state $[37,38]$ directly takes into account the influence of $p n$ interactions and yields significantly modified spectra for transitional nuclei in a nucleon pair approximation [39].

As already noted, the interest of the generalized seniority description lies both in its value as a simple description of the dominant BCS-like pairing correlations in a number-conserving framework and in its potential role as a foundation for bosonized descriptions of collective dynamics. However, setting these conceptual matters aside, it is also interesting to consider the shell-model dimensions involved in the cases for which the low generalized-seniority truncation would have the most practical utility directly, as a computational scheme. The present results are consistent with the expectation that this would be for a large single-particle space but sufficiently near a shell or subshell closure to restrict $p n$ correlations and deformation, as in the $\mathrm{Ca}$ and $\mathrm{Ti}$ isotopes. The Sn isotopes are the classic phenomenological example for a generalized seniority description [1]. As a concrete numerical example, consider the description of the light $\mathrm{Sn}$ isotopes $(Z=50)$, 
or adjacent $\mathrm{Cd}(Z=48)$ and $\mathrm{Te}(Z=52)$ isotopes, in the full $s d g$ major shell. The dimensions for the full shell-model spaces for various isotopic chains, including these, are shown in figure 9 , for $J=0$. In comparison, the generalized seniority $\left(v_{p}, v_{n}\right)=(2,2)$ space has the same size as the shell-model space for ${ }^{84} \mathrm{Mo}$ in these same orbitals dimension 427 for $J=0,1770$ for $J=2,2306$ for $J=4$, etc. The reduction in going from the full space to the generalized seniority $\left(v_{p}, v_{n}\right)=(2,2)$ space reaches ten orders of magnitude for ${ }^{104-106} \mathrm{Sn}$.

\section{Acknowledgments}

We thank F Iachello, S Pittel, J P Vary, S Frauendorf, and P Van Isacker for valuable discussions and M Horoi and B A Brown for generous assistance with NuShELLX. Dimensions for figures 2 and 9 were calculated using SU3SHELL [40]. This work was supported by the Research Corporation for Science Advancement under a Cottrell Scholar Award, by the US Department of Energy under Grant No. DE-FG02-95ER40934, and by a chargé de recherche honorifique from the Fonds de la Recherche Scientifique (Belgium). Computational resources were provided by the University of Notre Dame Center for Research Computing.

\section{References}

[1] Talmi I 1971 Nucl. Phys. A 1721

[2] Shlomo S and Talmi I 1972 Nucl. Phys. A 19881

[3] Gambhir Y K, Rimini A and Weber T 1969 Phys. Rev. 1881573

[4] Allaart K, Boeker E, Bonsignori G, Savoia M and Gambhir Y K 1988 Phys. Rep. 169209

[5] Iachello F and Arima A 1987 The Interacting Boson Model (Cambridge: Cambridge University Press)

[6] Otsuka T, Arima A and Iachello F 1978 Nucl. Phys. A 3091

[7] Iachello F and Talmi I 1987 Rev. Mod. Phys. 59339

[8] Gambhir Y K, Rimini A and Weber T 1971 Phys. Rev. C 31965

[9] Bonsignori G and Savoia M 1978 Nuovo Cimento A 44121

[10] Pittel S, Duval P D and Barrett B R 1982 Ann. Phys. (N.Y.) 144168

[11] Scholten O and Kruse H 1983 Phys. Lett. B 125113

[12] Bonsignori G, Savoia M, Allaart K, van Egmond A and te Velde G 1985 Nucl. Phys. A 432389

[13] Van Isacker P, Pittel S, Frank A and Duval P D 1986 Nucl. Phys. A 451202

[14] Navrátil P and Dobeš J 1988 Phys. Rev. C 372126

[15] Engel J, Vogel P, Ji X and Pittel S 1989 Phys. Lett. B 2255

[16] Lipas P O, Koskinen M, Harter H, Nojarov R and Faessler A 1990 Nucl. Phys. A 509509

[17] Otsuka T 1996 Prog. Theor. Phys. Suppl. 1255

[18] Yoshinaga N, Mizusaki T, Arima A and Devi Y D 1996 Prog. Theor. Phys. Suppl. 12565

[19] Monnoye O, Pittel S, Engel J, Bennett J R and Van Isacker P 2002 Phys. Rev. C 65044322

[20] Barea J and Iachello F 2009 Phys. Rev. C 79044301

[21] Sandulescu N, Blomqvist J, Engeland T, Hjorth-Jensen M, Holt A, Liotta R J and Osnes E 1997 Phys. Rev. C 552708

[22] Lei Y, Xu Z Y, Zhao Y M and Arima A 2010 Phys. Rev. C 82034303

[23] Caprio M A, Luo F Q, Cai K, Hellemans V and Constantinou Ch 2012 Phys. Rev. C 85034324

[24] Richter W A, van der Merwe M G, Julies R E and Brown B A 1991 Nucl. Phys. A 523325 
[25] Honma M, Otsuka T, Brown B A and Mizusaki T 2004 Phys. Rev. C 69034335

[26] Talmi I 1983 Prog. Part. Nucl. Phys. 927

[27] Caprio M A, Luo F Q, Cai K, Constantinou Ch and Hellemans V 2012 Generalized seniority in a major shell with realistic interactions Beauty in Physics: Theory and Experiment (AIP Conf. Proc. no 1488) ed Bijker R et al (Melville, New York: AIP) p 212

[28] Frank A and Van Isacker P 1982 Phys. Rev. C 261661

[29] Otsuka T 1993 Nucl. Phys. A 557 531c

[30] Mizusaki T and Ostuka T 1996 Prog. Theor. Phys. Suppl. 12597

[31] Chen J Q 1997 Nucl. Phys. A 626686

[32] Luo F Q and Caprio M A 2011 Nucl. Phys. A 84935

[33] Rae W D M computer code NuShellX (unpublished); Brown B A, Rae W D M, McDonald E and Horoi M, computer code NuSheLLX@MSU (unpublished)

[34] Sviratcheva K D, Draayer J P and Vary J P 2006 Phys. Rev. C 73034324

[35] Lei Y, Xu Z Y, Zhao Y M, Pittel S and Arima A 2011 Phys. Rev. C 83024302

[36] Talmi I 2001 Nucl. Phys. A 686217

[37] Maglione E, Catara F, Insolia A and Vitturi A 1983 Nucl. Phys. A 397102

[38] Pittel S and Dukelsky J 1983 Phys. Lett. B 1289

[39] Lei Y, Pittel S, Fu G J and Zhao Y M 2012 Spherical to deformed shape transition in the nucleonpair shell model (Preprint arXiv:1207.2297v1)

[40] Dytrych $\mathrm{T}$ et al computer code Su3shell (unpublished) 